PS2-22:

Geisinger Health System's Clinical Interventions for Preventing Pediatric Obesity - Infancy through School-age

Lisa Bailey-Davis ${ }^{1}$; Rebecca Stametz'; William Cochran ${ }^{1}$; Megan Mariotti ${ }^{1}$; Jonathan Darer ${ }^{1}$; Gloria Gerrity ${ }^{1}$; Xiaowei Yan ${ }^{1}$; Joseph Leader ${ }^{1}$; Leann Birch $^{2}$; Jennifer Savage Williams ${ }^{2}$; Gregory Welk ${ }^{3}$

${ }^{1}$ Geisinger Health System; ${ }^{2}$ Pennsylvania State University; ${ }^{3}$ Iowa State University

Background/Aims: Childhood obesity remains a priority issue for the nation and health systems yet little is known about the effectiveness of pediatric obesity prevention interventions overall and evidence is particularly lacking with regard to prevention in clinical settings. Our aim is to discuss process steps in developing two clinical interventions to prevent obesity and their research designs to evaluate effectiveness. Methods: Two interventions were developed, the first to address early childhood obesity prevention among 0-26 month old children and the second to address prevention among children aged 2-9 years, with intentional overlap to coordinate care. A team, comprised of pediatric clinical operations, clinical innovations, research, eHealth, biostatistics, and external university partners, worked collaboratively to develop each intervention and their respective research designs. Results: Each intervention is delivered at scheduled Well Child Visits with consistent components: 1) parent self-reported data to screen for parenting practices, child behaviors, and home environments; 2) parent engagement via screening and risk prioritization; 3 ) best practice alerts for providers to aid analytical assessment of risk; 4) primary care provider talking points and a SmartSet of parent educational materials; 5) electronic health record progress note construction; and 6) post-visit education. A cluster randomized design is being used to evaluate the effectiveness of both interventions with 6 clinics assigned to the intervention and 6 matched clinics providing standard care. Process outcomes including intervention fidelity and parent engagement are being examined as well as health outcomes. Data are being collected and will be compared to 12-month indicators to evaluate changes in child weight/ length or BMI-for-age and BMI; child behaviors; and parenting practices. Conclusions: A focus on health system goals, a priority health issue, and principles of the learning health care system engaged internal and external entities to leverage resources and successfully develop and implement pediatric obesity prevention interventions. In addition, two pragmatic trials to examine the implementation and effectiveness of the interventions are underway and will contribute to a gap in the evidence base.

Keywords: Clinical intervention; Prevention

doi:10.3121/cmr.2014.1250.ps2-22

\section{Chronic Conditions/Diabetes/Obesity}

A2-1:

Longitudinal eGFR, eGFR Slope, and Cardiovascular Events Among Adults with CKD

H. Lester Kirchner ${ }^{1}$; Xiaoqin Tang ${ }^{1}$; Joseph Leader ${ }^{1}$; Robert Perkins ${ }^{2}$

${ }^{1}$ Geisinger Health System; ${ }^{2}$ Bassett Medical Center

Background/Aims: Single time-point GFR estimates are used for risk prognostication among those with CKD. Various applications of longitudinal eGFR using linear models, including time-independent slope and variability, have been shown to independently predict cardiovascular events. We wished to extend these applications by modeling longitudinal eGFR-forming estimates of current eGFR value and time-dependent eGFR slope at any point in time-and then test the independent association with cardiovascular event risk in an adjusted, joint longitudinal-survival model. Methods: We retrospectively assembled a cohort of Geisinger primary care patients with stage G3-G4 CKD(1/1/2001 to 6/30/2012) who had a minimum of 4 outpatient eGFR results and 6-months follow-up. Exclusion criteria included a history of dialysis or renal transplantation. Patients were followed through 12/31/2012 for the first occurrence of MI, HF, or stroke (CVD event), or were censored at ESRD, death or end of study. EGFR was modeled using a random intercept and slope model with a flexible spline transformation of time to capture non-linear trends. Time to first CVD event post-CKD diagnosis was modeled using a Cox Proportional Hazard regression model.
The longitudinal and survival models were then jointly estimated. Results: A total of 24,777 patients met cohort entry criteria. A random sample of $90 \%$ $(22,300)$ was used for model development (mean age $69.3 \mathrm{y}, 42 \%$ (9409) male, $28 \%$ (6277) with diabetes, mean baseline eGFR $51 \mathrm{~mL} / \mathrm{min}$ ) and contributed 119,165 person-years of follow up. 6\% (1339) had a prior CVD event. During follow-up $4216(19 \%)$ patients had a post-CKD CVD event. In fully adjusted models, each $5 \mathrm{~mL} / \mathrm{min} / 1.73 \mathrm{~m} 2$ decrease in current eGFR was associated with a hazard ratio (HR) of 1.05 (95\% CI 1.04, 1.06). For the timedependent slope of eGFR trend, each $2 \mathrm{~mL} / \mathrm{min} / 1.73 \mathrm{~m} 2 /$ year decrement was associated with a HR of $1.06(95 \%$ CI 1.03, 1.10). Both diabetes and gender modified the eGFR slope associations, such that the associations were significantly greater among non-diabetics $(H R=1.17)$ and females $(H R=$ 1.11). Conclusions: Longitudinally modeled eGFR and time-dependent eGFR slope each independently associates with CVD risk among patients with moderate and advanced CKD. The relative prognostic value of static, single time point eGFR vs. joint modeling of eGFR warrants further investigation.

Keywords: Chronic kidney disease; Joint longitudinal-survival models doi:10.3121/cmr.2014.1250.a2-1

\section{A2-2: \\ Improving Diabetes Management with Mindfulness-based Stress Reduction}

Robin Whitebird'; Mary Kreitzer²; Gabriela Vazquez-Benitez'; Christopher Enstad $^{1}$; Logan Stuck ${ }^{1}$; Patrick O'Connor ${ }^{1}$

${ }^{1}$ HealthPartners; ${ }^{2}$ University of Minnesota

Background/Aims: Managing diabetes can be challenging and stressful for many people resulting in poor diabetes control and increased mental health issues such as depression and anxiety. Methods: We conducted a pilot study of mindfulness-based stress reduction (MBSR), a combination of mindfulness meditation and gentle yoga, to look at whether it could help patients better manage their diabetes. We recruited 38 people with diabetes and $2 \mathrm{HbA} 1 \mathrm{c}$ values $>8$ in the prior 18 months to complete a community-based MBSR course. Participants attended eight-weekly intervention sessions and participated in home-based MBSR practice. Surveys and HbA1c values were obtained at pre and post-intervention, Cohen's-d statistic was estimated for survey outcomes. Pre-post change was evaluated using paired t-test. Results: Participants were 31- to 78-years-old $(M=57)$, the majority were female (68 $\%$ ), white (70\%), employed, with some college education. Mean $\mathrm{HbAlc}$ preintervention was 9.18. Participants showed significant improvement in prepost measures of HbA1c (change $.73 \%, P=.000$ ), overall mental health (Cohen's-d .69, $P=.001$ ), stress (Cohen's-d -.76, $P=.001$ ), depression (Cohen's-d .62, $P=.001$ ), and anxiety (Cohen's-d .66, $P=.001$ ). There was also improvement in two measures of diabetes management: Problem Area in Diabetes Questionnaire (Cohen's-d -.71, $P=.002$ ) and the Diabetes Empowerment Scale (Cohen's-d .80, $P=.000$ ). Conclusions: These results suggest that MBSR may offer a safe and effective method for helping people better manage diabetes and improve their mental health. Effect sizes were large and significant pre-post differences were found indicating that a larger clinical trial is warranted.

Keywords: Diabetes; Complementary therapies doi:10.3121/cmr.2014.1250.a2-2

\section{A2-3:}

Impact of Mild Chronic Kidney Disease Stage on Outcomes after Total Hip or Knee Arthroplasty

Jove Graham ${ }^{1}$; Brian Deegan'; Thomas Bowen'; Raveesh Richard'; Robert Perkins $^{2}$; Michael Foltzer ${ }^{1}$

${ }^{1}$ Geisinger Health System; ${ }^{2}$ Bassett Healthcare

Background/Aims: Dialysis and kidney transplantation adversely impact outcomes of total hip or knee arthroplasty (THA, TKA), but complication rates have not been reported for patients with less advanced stages of chronic kidney disease (CKD). Surgeons lack the data necessary to have informed discussions regarding anticipated outcomes of joint replacement for these patients. Methods: We retrospectively reviewed electronic health records of 779 adults with stages 1,2, and 3 CKD not requiring dialysis or transplantation 
who underwent THA or TKA from 2004-2011, to assess infection, revision, 90-day readmission and mortality rates. Patients with less than 12 months follow-up, open fracture, prior joint surgery, pregnancy, or acute kidney injury were excluded. Chi-square analysis and Cox survival analysis compared these outcomes between the stage 1-2 and stage 3 groups, stratified by joint replaced (THA vs TKA). All models adjusted for age, sex and BMI at surgery. Results: No statistically different rates of revision or infection between Stage 3 vs. Stages 1-2 were seen, although there was a trend toward increased infections in Stage 3. THA patients with Stage 3 showed a significantly increased mortality rate compared to Stage 1-2 THA patients (HR 3.40, 95\% CI $=1.25-9.23, P=0.02$ ). Conclusions: $\mathrm{CKD}$ affects nearly $15 \%$ of the U.S. population many of whom undergo joint replacement. End stage kidney disease (patients post-transplant or on hemodialysis) has been consistently associated with increased rates of infection and revision in excess of our observed outcomes, but the overall rate of infection/revision in our study population was only slightly higher than reported rates in the general population $(2-7 \%$ vs. $1-2 \%$, respectively). CKD should not preclude joint replacement, but these data can help clinicians engage in meaningful informed discussions with patients with mild kidney disease regarding risks for infection, revision and death following joint replacement

Keywords: Chronic kidney disease; Total joint replacement

doi:10.3121/cmr.2014.1250.a2-3

A2-4:

Declining Rates of Major Cardiovascular Events in Adults With and Without Diabetes: 2005-2011

Jay Desai ${ }^{1}$; Gabriela Vazquez Benitez ${ }^{1}$; Emily Schroeder ${ }^{2}$; Gregory Nichols ${ }^{3}$; John Steiner ${ }^{2}$; Katherine Newton ${ }^{4}$; Ram Pathak ${ }^{5}$; Renuka Adibhatla ${ }^{1}$; Zhiyuan $\mathrm{Xu}^{1}$; Mary Becker'; Patrick O'Connor ${ }^{1}$

${ }^{1}$ HealthPartners; ${ }^{2}$ Kaiser Permanente Colorado; ${ }^{3}$ Kaiser Permanente Northwest; ${ }^{4}$ Group Health Research Institute; ${ }^{5}$ Marshfield Clinic Research Foundation

Background/Aims: A major goal of diabetes care is the prevention of major cardiovascular events (CVE). Its success can be measured by quantifying trends over time in major CVE in adults with and without diabetes. To quantify trends in occurrence of major CVE in adults with and without diabetes who received care at 11 U.S. managed care organizations from 2005 to 2011. Methods: Study subjects included 2.4 million diabetes and nondiabetes subjects, frequency matched by age and gender $(10,000,000$ personyears). Clinical and demographic data were extracted from EMR at each site. Major CV events were identified based on primary discharge diagnoses from inpatient encounters for myocardial infarction/acute coronary syndrome (MI/ ACS), stroke, and chronic heart failure (CHF). Age and sex-adjusted to the 2010 US census population even rates over time were computed. Generalized linear models were used to evaluate the significance of observed differences CVE trends in defined groups of subjects. Results: Mean age at cohort entry was 59 years $(\mathrm{SD}=14), 48 \%$ female, and $46 \%$ Whites. Rates per 1000 of CVE in 2010 for diabetes subjects were (MI/ACS: 4.4, stroke 4.0, CHF 6.9); event rates were more than twice the rate of non-diabetes subjects. A 5-year decline of $32 \%$ was observed in diabetes subjects and $27 \%$ in non-diabetes subjects for MI/ACS. Similar decline was observed by gender and age groups. Conclusions: Between 2005 and 2011, rates of major cardiovascular events (CVE) declined significantly among adults with diabetes. Decline was greater in patients with diabetes, likely associated with improvements in diabetes care.

Keywords: Diabetes; Chronic disease

doi:10.3121/cmr.2014.1250.a2-4

A2-5:

Addressing Missing Data and Clinical Relapse Can Improve Composite Diabetes Quality of Care Measure

Patrick O'Connor ${ }^{1}$; Jay Desai ${ }^{1}$; JoAnn Sperl-Hillen ${ }^{1}$; Renuka Adibhatla ${ }^{1}$; Mary Becker ${ }^{1}$; Kris Ohnsorg ${ }^{1}$; Gabriela Vazquez Benitez ${ }^{1}$

${ }^{1}$ HealthPartners

Background/Aims: To identify strategies likely to improve composite measures of diabetes care quality. Methods: Study subjects were 14,750 adults with diabetes identified in one care delivery system in the SUPREMEDM project (HS19859) who had BP measures in both 2005 (Y1) and 2010 (Y2). We report the proportion of patients who met clinical goals (A1c $<8 \%$, LDL $<100 \mathrm{mg} / \mathrm{dl}$, BP $<140 / 90$, no tobacco use, or composite D4 goal requiring all 4 components to be at goal) in $\mathrm{Y} 1$ but not in $\mathrm{Y} 2$, based on the last available assessments in each of the two 12-month periods. Results: Among those with measures in $\mathrm{Y} 1$ and $\mathrm{Y} 2$ and at goal in $\mathrm{Y} 1$, the proportion not at goal in $\mathrm{Y} 2$ for A1c, BP, LDL, tobacco, and the D4 was $13.7 \%, 8.5 \%$, $12.8 \%, 1.6 \%$, and $20.7 \%$ respectively. When those missing measures in Y2 were classified as not at goal in Y2, the proportion of those in control in Y1 but not in Y2 for A1c, BP, LDL, tobacco, and the D4 was $25.7 \%, 8.5 \%$, $27.1 \%, 24.1 \%$, and $52.6 \%$ respectively. Among those with measures in both $\mathrm{Y} 1$ and $\mathrm{Y} 2$, the proportion of all those not at goal in year 2 who had been at goal in Y1 for A1c, BP, LDL, tobacco and the D4 was $21.1 \%, 45.2 \%, 25.9 \%$, $14.1 \%$, and $14.7 \%$ respectively. The proportion of those who failed to meet the $\mathrm{D} 4$ goal because of missing measures of one or more $\mathrm{D} 4$ components was $52.2 \%$ in $\mathrm{Y} 1$ and $54.1 \%$ in $\mathrm{Y} 2$. Conclusions: Strategies to improve composite measures of diabetes quality include: (a) Record measures of A1c, LDL, and tobacco use more frequently, and (b) Implement strategies to prevent clinical relapse in those at highest risk of relapse, especially for BP. Keywords: Diabetes; Quality of care

doi:10.3121/cmr.2014.1250.a2-5

D2-2:

How Did Pharmacists Help Patients Achieve Blood Pressure Control in a Randomized Trial of Home Blood Pressure Telemonitoring Plus Pharmacist Management?

Karen Margolis ${ }^{1}$; Stephen Asche ${ }^{1}$; Anna Bergdall ${ }^{1}$; Sarah Groen ${ }^{1}$; Krissa Klotzl $^{1}$; Ryan Michels ${ }^{1}$; Daniel Rehrauer'; JoAnn Sperl-Hillen'; Nicole Trower'; Patrick O’Connor ${ }^{1}$

\section{${ }^{1}$ HealthPartners}

Background/Aims: The Hyperlink trial tested a 12-month intervention combining home blood pressure (BP) telemonitoring with pharmacist case management in patients with uncontrolled hypertension. The intervention resulted in improved BP control compared to usual care at both 6 and 12 months $(57 \%$ vs. $30 \%, P=0.001)$. The mean number of medication classes increased compared to usual care $(0.63$ vs. $0.22, P<0.001)$. We use process of care data recorded by the pharmacists and BP telemonitoring data to elucidate reasons for intervention success. Methods: Hyperlink randomized 450 patients with uncontrolled BP from 16 primary care clinics, and 228 intervention group patients were enrolled from clinics. They used a home blood pressure telemonitor to transmit BP readings to a study pharmacist. Following an in-person intake visit, pharmacists consulted with patients over the phone every 2-4 weeks during the first 6 months, and at 2 month intervals thereafter. They adjusted antihypertensive therapy using a specific treatment algorithm based on BP telemonitoring data. The home BP goal was $<135 / 85$ $\mathrm{mm} \mathrm{Hg}(<125 / 75$ for patients with diabetes or kidney disease.) Results: Mean BP at the research clinic enrollment visit was 148/85, and 143/85 at the pharmacist intake visit. At this visit, pharmacists changed the antihypertensive drug regimen for $10 \%$ of patients, while medication changes occurred for $33 \%$ at phone visit $1,36 \%$ at phone visit 2 , and $19 \%$ at phone visit 3 . Thereafter, medication changes continued to decline. At the intake visit, pharmacists judged medication adherence to be high $(>80 \%)$ in only about half of patients, but at subsequent phone visits in $>90 \%$ of patients. The mean home BP recorded by the pharmacists for patients at the first phone visit was $136 / 80$, and fell steadily to a mean of $126 / 74$ at 3 months, and thereafter declined more slowly to $123 / 73$ at 5 months, with little change thereafter. Conclusions: Improved adherence and appropriate adjustments to the antihypertensive treatment regimen based on home BP telemonitoring resulted in rapid lowering of BP over a 3-month period. Our results imply that intensive intervention may only be needed for about 3 months in many patients with uncontrolled hypertension.

Keywords: Hypertension; Team-based care

doi:10.3121/cmr.2014.1250.d2-2 